\title{
Contributions of the human pulvinar to linking vision and action
}

\author{
SHAI DANZIGER \\ Ben Gurion University of the Negev, Beer Sheva, Israel \\ and \\ ROBERT WARD, VANESSA OWEN, and ROBERT RAFAL \\ University of Wales, Bangor, Wales
}

\begin{abstract}
In 3 patients with unilateral pulvinar lesions, we tested the pulvinar's role in selective attention processing. Each patient completed four variants of a flanker interference task in which they reported the color of a square of a specified size while ignoring an irrelevant flanker that appeared either contralesionally or ipsilesionally to the target. The main finding was that when target location was not known and target and flanker were associated with competing responses, reaction times to contralesional targets were longer than those to ipsilesional targets. Our findings suggest that pulvinar damage produces a contralesional deficit in response competition.
\end{abstract}

Attention mechanisms provide humans the adaptive ability to selectively process goal-relevant information while suppressing irrelevant information. Recent neurobiological theories have postulated that a distributed neural network mediates attentional selection. This network is thought to include cortical areas such as the parietal cortex (PAC) and prefrontal cortex (PFC) as well as subcortical structures including the superior colliculus and the pulvinar nucleus of the thalamus (Posner \& Petersen, 1990; Rafal \& Posner, 1987; Sturm et al., 1999). The aim of this study was to investigate the contribution of the pulvinar to selective processing by assessing the performance of patients with pulvinar lesions on a response competition task that taps several components of attention.

Although there is a growing body of research on the role of the pulvinar from single-cell recording (Petersen, Robinson, \& Morris, 1987; Robinson \& Petersen, 1992) and reversible lesion studies in nonhuman primates (Desimone, Wessinger, Thomas, \& Schneider, 1990; Petersen, Robinson, \& Morris, 1987), only a few studies have examined the pulvinar's role in selective attention in humans. Of these, several have investigated spatial orienting performance in pulvinar-lesioned patients (Experiment 1 of Danziger, Ward, Owen, \& Rafal, 2002; Rafal \& Posner, 1987; Sapir, Rafal, \& Henik, 2002), one has investigated feature binding in pulvinar-lesioned patients (Ward, Danziger, Owen, \& Rafal, 2002), and two brain-imaging studies have examined the role of the healthy pulvinar in

The experiments reported herein were conducted at the University of Wales at Bangor. Correspondence concerning this article should be addressed to S. Danziger, Ben Gurion University of the Negev, Beer Sheva, P. O. Box 643, Israel (e-mail: shaidanz@bgumail.bgu.ac.il). attentional selection (LaBerge \& Buchsbaum, 1990; Morris, Friston, \& Dolan, 1997).

The main finding emerging from the spatial orienting studies is that pulvinar-lesioned patients are slower to detect or report the location of a contralesional target relative to an ipsilesional target. The typical procedure that has been used in these studies is to present a lateralized precue that is temporally followed by a lateralized target to which subjects respond. Comparisons are then made between conditions in which the cue and target share or do not share the same spatial location. In Rafal and Posner's (1987) study, for example, the target appeared at the cued location on $80 \%$ of the trials and at the noncued location on the remaining trials. At each of four cue-target stimulus onset asynchronies (SOAs) ranging from 50 to $1,000 \mathrm{msec}$, the performance of 3 patients was characterized by longer reaction times (RTs) to contralesional targets regardless of whether attention had been cued contralesionally or ipsilesionally. This finding was advanced as evidence that pulvinar damage produces a deficit in engaging attention contralesionally (Posner \& Petersen, 1990). In Posner and Petersen's framework of attentional orienting, the engage operation was distinguished from a move operation thought to be mediated by midbrain structures and a disengage operation thought to be under parietal control (Friedrich, Egly, Rafal, \& Beck, 1998; Morrow \& Ratcliff, 1988; Posner, Walker, Friedrich, \& Rafal, 1984).

In the PET studies of LaBerge (1990) and LaBerge and Buchsbaum (1990), healthy subjects identified a lateralized visual target that appeared either alone or with distractors present in the same hemifield. LaBerge and Buchsbaum found increased pulvinar activation when the contralateral visual field contained distractors relative to when it did not. They interpreted this result as ev- 
idence that the pulvinar contributes to selective attention processes by both facilitating target processing and inhibiting the processing of distractors.

More recently, Morris et al. (1997) measured brain activation as a function of the behavioral salience of stimuli. Changes in behavioral salience were induced through adverse conditioning. During the initial conditioning phase of the experiment, presentations of emotionally expressive faces were paired either with an aversive white noise stimulus or with silence. During subsequent attentive viewing of faces, the adversely conditioned faces were associated with greater activation in the right pulvinar as well as in other right-hemisphere structures including the orbitofrontal cortex, the superior frontal gyrus, and other thalamic activation. Morris et al. claimed that the observed increase in pulvinar activity reflects increased attention to stimuli with greater salience for action. In that study, targets were presented singly at 15 -sec intervals so that demands on selective attention were minimal and there was no filtering requirement.

In summary, the aforementioned research suggests an important role for the pulvinar in engaging attention, selecting targets from cluttered areas, and possibly selecting or tagging salient stimuli. Importantly, in none of the mentioned studies was the pulvinar's role in response selection examined save for the study by Danziger et al. (2002), in which 2 patients were asked to identify targets that appeared at one level of a global-local hierarchical stimulus while ignoring a distractor present at the other level. In this task, the distractor indicated either the same response as did the target or a different response. RTs to targets in both visual fields were similar, as were interference effects from the ignored distractors. In other words, there was no evidence for an engage deficit or impairment in target selection in this response competition task.

In the present study, our goal was to further test the pulvinar's role in engaging visual attention, in filtering distractors, and in response activation and selection, by using another much-studied response competition task. Here, we examined the effects of unilateral pulvinar lesions on selective attention by using a flanker interference paradigm that requires speeded responses to a target in the presence of flankers (Eriksen \& Yeh, 1985). This paradigm has been widely used to study selective attention processing in both healthy individuals (Coles, Gratton, Bashore, Eriksen, \& Donchin, 1985; Diedrichsen, Ivry, Cohen, \& Danziger, 2000; Eriksen \& Yeh, 1985; Lavie, 1995; Miller, 1991) and patients with cortical damage (Cohen, Ivry, Rafal, \& Kohn, 1995; Rafal et al., 1996; Ro, Cohen, Ivry, \& Rafal, 1998).

In a typical flanker task, subjects make speeded responses to a target appearing at a known location while ignoring flanking stimuli that appear on either side of the target and at varying distances from it. The stimulus set typically consists of two or more possible targets that are assigned to different response categories. Flankers can be either congruent with the target, indicating the same response as that for the target (e.g., when subjects are re- porting color, the target and flanker are both red) or incongruent with the target, indicating a response different from that for the target (e.g., a red target and a green flanker). In most cases, choice responses to the target are faster and more accurate in the congruent condition than in the incongruent condition. The RT difference between congruent and incongruent conditions has been labeled the flanker congruency effect (FCE). The presence of an FCE indicates that flankers have been processed at least to the level of response activation.

Rafal and colleagues (Cohen et al., 1995; Rafal et al., 1996; Ro et al., 1998) have previously employed a single flanker version of the flanker paradigm, in which a single flanker appears either ipsilesionally or contralesionally to the target, to examine the effects of both parietal and prefrontal lesions on attentional selection. The use of a single flanker allows two forms of interference to be separately assessed. First, the size of the FCE can be compared for conditions in which the flankers are either contralesional or ipsilesional to the target. An exaggerated FCE from contralesional flankers would suggest a deficit in filtering or inhibiting irrelevant information, whereas a reduced FCE from contralesional flankers would suggest that the lesioned structure is involved in the activation of response codes that are thought to produce the FCE. Second, regardless of whether the flanker is associated with a congruent or incongruent response code, the overall latencies to targets can be compared on the basis of the relative positions of the target and flanker. This provides a measure of selective attention bias, or the strength with which contralesional and ipsilesional items compete for selective attention. For example, an overall slowing when flankers are ipsilesional rather than contralesional to the target suggests that ipsilesional stimuli capture attention more readily (i.e., are more readily engaged) than do contralesional ones and/or are more difficult to disengage from (Petersen, Robinson, \& Morris, 1987; Rafal \& Posner, 1987).

\section{GENERAL METHOD}

\section{Patients}

All 3 patients in this study had been neurologically intact prior to sustaining a hypertensive hemorrhage centered in the posterior thalamus. Participation in these experiments began 2-5 months after the initial hemorrhage and after the patients provided oral and written consent. The research and consent procedures were approved by the NHS ethics committees of the participating hospitals. In all cases, the patients had returned to independent living with some residual motor or sensory deficits, but they had no mental symptoms compromising their functions of daily life and social interactions. At the time of testing, all 3 patients had intact visual fields and did not have signs of hemispatial neglect or visual extinction. An MRI was done during the time of testing, and 1-mm sections in the axial, coronal, and sagittal planes were obtained through the thalamus. The residual lesion involved the posterior lateral nucleus of the thalamus in all patients and, in all, extended to involve the pulvinar nucleus. Figure 1 shows a composite neuroimage reconstruction of the lesions of all 3 patients (MRIcro software; available at www.mricro.com).

G.J. is a 54-year-old, right-handed man who suffered a hematoma centered in the left thalamus. Examination at the time of testing 

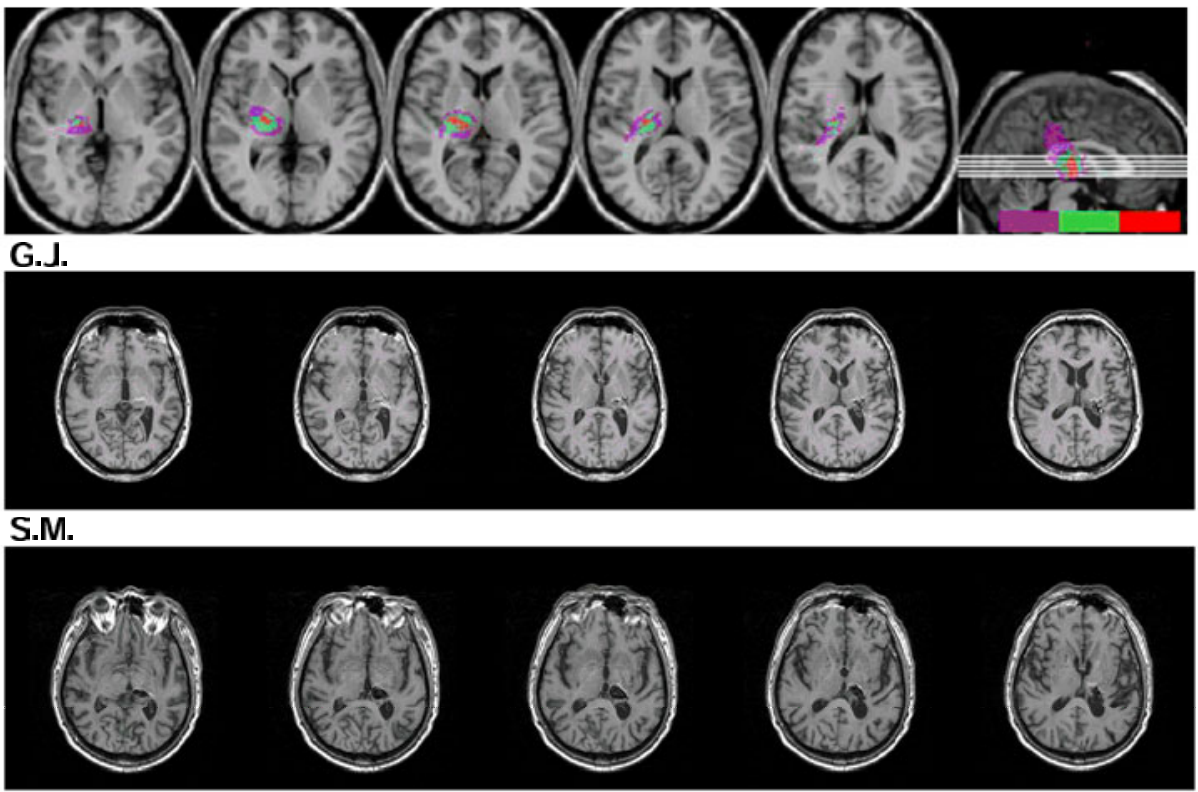

Figure 1. Composite MRI reconstruction of the lesions of all 3 patients. Each patient had a T1 MRI with 1-mm sections and a reconstruction generated on a normalized brain using MRIcro software (available at http://www.mrc-cbu.cam.ac.uk/personal/chris.rorden/mricro.htm). The lines on the lateral view on the right indicate the levels of the axial sections. The color scale indicates the number of patients with a lesion in the area indicated. (Red indicates the area involved by the lesion in all 3 patients; green, the area involved in 2 of the 3 patients; and purple, the area involved by only 1 of the 3 patients.) The entire pulvinar was destroyed in Patient S.M.; the least amount of pulvinar was damaged in Patient T.N., in whom pulvinar involvement was limited to the most anterior and dorsal part of the nucleus (see Ward et al., 1999).

3 months later showed only slight weakness of the right hand and arm and increased stretch reflexes in the right fingers and wrist. He was able to oppose his right thumb to the 5th finger. There was dense sensory loss to all modalities (touch, temperature, pin, position, and vibration) in the right hand; these deficits were also severe in the proximal arm and leg although with some recovery of position sense proximally. He was able to walk with a cane. The MRI scan showed the lesion to be centered in the left lateral pulvinar and posterior lateral thalamic nuclei.

S.M. is a 61-year-old, right-handed, hypertensive man who suffered a hematoma centered in the left pulvinar. Examination at the time of testing 2 months later revealed mild weakness of the right hand but somewhat greater weakness of the shoulder and the tendon. Stretch reflexes were normal. Light touch, pin, and temperature sensation were intact. Stereognosis was very poor in the right hand. Position sense was impaired in the right hand, although he could determine the direction of finger movement for large excursions. He was able to walk with a cane. The MRI scan showed almost complete destruction of the pulvinar nucleus.

T.N. is a 54-year-old, right-handed, hypertensive woman who suffered a hematoma centered in the right thalamus 5 months prior to initial testing. Examination during testing revealed weakness and hyperactive reflexes in the left arm and leg. She could move her fingers but could not oppose the thumb to the 5 th finger. Pin sensation was present but less intense on the left. Temperature sensation seemed equivalent bilaterally. Position sense was absent in the fingers and was less severely impaired at the wrist, elbow, and shoulder. There was pseudoathetosis of the left hand, and when her eyes were closed, her left arm drifted and she was not aware of its position. Vibration sense was present on the left but was diminished in comparison with the right. She was able to walk with a cane. The MRI scan showed the lesion to involve the posterior lateral thalamic nucleus on the right and to extend dorsally into the most latero-anterior and dorsal part of the pulvinar nucleus (see Figure 1).

\section{General Task Procedures}

In each of the four experiments, the patients responded to stimuli presented on a laptop computer. On each trial of the various experiments, they were shown two colored squares that differed in size. One square was positioned at the center of the display, and the other was located in either the ipsilesional or the contralesional field. The patients were asked to report the color of the smaller square and to ignore the larger square that served as a flanker. The target square was colored either red or green, and the flanker square was either the same color as the target (coding for the same response) or, with equal probability, the other color (coding for a response incongruent with a correct response). ${ }^{1}$ Both stimuli remained on the screen until a response was made.

In the first three experiments, the patients responded by pressing one of two keys on the laptop keyboard that were arbitrarily as- 
signed to each of the two possible color responses. In Experiment 1, the target location was predictable, appearing always in the center, and the flanker appeared either near or far from the target. In Experiments 2 and 3, the target was equally likely to appear in the center or in either periphery. With central targets, the flanker appeared in either the contralesional or ipsilesional field; with peripheral targets, the flanker appeared centrally, at fixation. Experiment 3 was identical to Experiment 2, except that the flankers were either neutral or incongruent with respect to the target response. In Experiment 4, the target appeared, unpredictably, in either the contralesional or ipsilesional field, and the flanker always appeared in the center. In this experiment, the patients responded vocally to the target, so maintaining arbitrary stimulus-response mappings was not required.

\section{EXPERIMENT 1 Central Report Task With Manual Responses}

In Experiment 1, we investigated whether patients with pulvinar damage would show different FCEs for the presentation of contralesional as opposed to ipsilesional flankers. If pulvinar damage results in a deficit in filtering or inhibiting response activation from contralesional flankers, then the FCE should be larger on contralesional than on ipsilesional flanker trials. In contrast, if pulvinar damage weakens response activation from contralesional flankers, then the FCE should be smaller on contralesional than on ipsilesional flanker trials. This experiment also tested for a selective attention bias against the contralesional field (Rafal \& Posner, 1987). If pulvinar damage makes it difficult to engage relatively contralesional stimuli, as has been argued by Rafal and Posner and as has been demonstrated in patients with hemispatial neglect (Cohen et al., 1995), we would expect longer RTs to targets flanked by ipsilesional flankers than to those flanked by contralesionalflankers. As a further test of the role of the pulvinar in attentional selection, we manipulated the distance between the target and the flanker. In healthy human observers, FCEs decrease as the targetflanker distance increases (Miller, 1991). On the basis of the observations of LaBerge (1990) and LaBerge and Buchsbaum (1990), who found increased pulvinar activation in a PET imaging study with healthy humans only when the target was flanked by many distractor stimuli, we postulated that pulvinar damage would affect performance far more in the near than in the far flanker condition.

\section{Method}

Apparatus and Stimuli. Stimulus presentation and response collection were controlled by either a Macintosh PowerBook 3400c or a Macintosh PowerBook G3. The target stimulus was a small solid square $\left(0.64^{\circ} \times 0.64^{\circ}\right)$, colored either red or green, that appeared at fixation. The flanker was a larger solid square $\left(0.93^{\circ} \times\right.$ $0.93^{\circ}$ ), colored either red or green, that appeared to the left or to the right of the target. A small black cross (Chicago font, point size 12) served as a fixation point, and the background was white.

Procedure. Each patient completed multiple blocks of trials in two or three sessions. In each session, the computer monitor was placed at a viewing distance of approximately $80 \mathrm{~cm}$. The patients responded to the target color by pressing the "V" key on the keyboard for red targets and the "N" key for green targets. Patients G.J. and S.M. used the index finger of the ipsilesional hand to press the two keys, whereas Patient T.N. used the index and middle fingers of the ipsilesional hand to press the two keys. The patients were instructed to maintain fixation and as quickly and as accurately as possible to respond to the target while ignoring the flanker. A trial began with a fixation point appearing in the middle of the monitor. After $500 \mathrm{msec}$, a central target and a flanker that appeared either to the left or to the right of the target replaced the fixation point. Both stimuli remained visible until a response was made or else terminated after 10,000 msec. Each trial began 1,000 msec after the previous trial ended. In near blocks, the distance between the centers of the target and flanker was $1.36^{\circ}$. In far blocks this distance was $4.5^{\circ}$

Each patient completed multiple blocks of 92 trials that alternated between near and far flanker blocks. Within each block, half of the trials were congruent (e.g., a red target flanked by a red flanker), and half were incongruent (e.g., a red target flanked by a green flanker). On half of the trials the flanker appeared contralesionally, and on half it appeared ipsilesionally. Target color, flanker color, and flanker field were each selected with equal probability, at random and combined orthogonally.

\section{Results}

The RT analysis included only correct response trials. Failures to respond in a speeded manner (latencies longer than $3,000 \mathrm{msec}$ ), anticipatory responses (latencies shorter than $100 \mathrm{msec}$ ), and RTs greater than 3 standard deviations from each individual's mean were excluded. For each patient, these accounted for less than $2 \%$ of the trials. Errors accounted for less than $2.1 \%$ of responses for each of the patients.

In each experiment, mean RTs were calculated for each experimental condition for each subject for an entire session. For the group analysis, these means were entered into a three-way (congruency-congruent and incongruent; flanker field - contralesional and ipsilesional; and distance - near and far) repeated measures analysis of variance (ANOVA) with subject as the random variable. For the individual subject analysis, means were calculated for each experimental condition in each block. The resulting means were entered into the three-way repeated measures ANOVA with block as the random variable. Table 1 shows the mean RTs for each patient for all four experiments. Figure 2 shows the group RTs for congruent and incongruent trials as a function of flanker field and distance.

There were no significant main effects or interactions in the group analysis at the .05 level. We also performed analyses of individual patients. For Patients T.N. and S.M., RTs on congruent trials were significantly shorter than those on incongruent trials [T.N., $F(1,8)=17.7$, $p<.005$; S.M., $F(1,3)=19.1, p<.05]$. This effect approached significance for Patient G.J. $[F(1,5)=6.2, p=$ $.054]$. Although the distance $\times$ congruency interaction was not significant at the group level $[F(1,2)=8.76$, $p=.098]$, Table 1 shows that RT differences between congruent and incongruent trials were numerically larger in the near distractor blocks than in the far distractor blocks for each patient in each field. This pattern is similar to that found with healthy subjects. More important, Table 1 also shows that, for all patients, FCEs were similar in the contralesional and ipsilesional flanker conditions 
Table 1

Mean Response Times (RTs, in Milliseconds) With Percentages of Errors (PEs) for Each Patient in Each Experiment

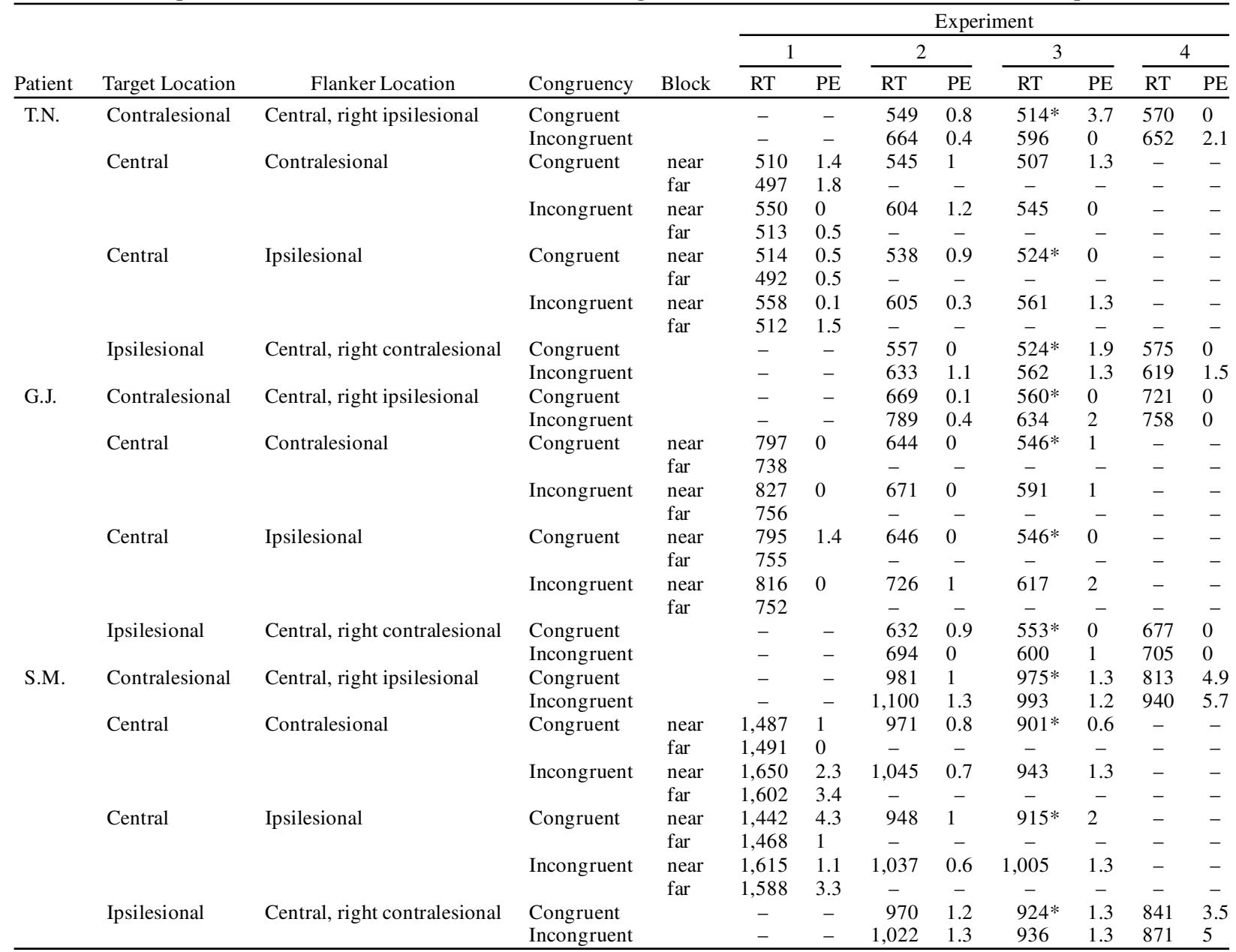

Note-Data are shown as a function of target location (contralesional, central, and ipsilesional), flanker location (central, relatively ipsilesional, and relatively contralesional), and congruency (congruent and incongruent). Patient T.N. performed 9 blocks in Experiment 1, 6 blocks in Experiment 2, 10 blocks in Experiment 3, and 10 blocks in Experiment 4. Patient G.J. performed 6 blocks in Experiment 1, 7 blocks in Experiment 2, 12 blocks in Experiment 3, and 6 blocks in Experiment 4. Patient S.M. performed 4 blocks in Experiment 1, 9 blocks in Experiment 2 , 10 blocks in Experiment 3, and 6 blocks in Experiment 4. *The neutral condition, which replaced the congruent condition in Experiment 3.

$\left(F_{\mathrm{S}}<1\right.$ for each patient) and that RTs to a relatively contralesional target (flanker appeared in the ipsilesional field) did not differ from those to a relatively ipsilesional target (flanker appeared in the contralesional field).

\section{Discussion}

The most striking finding of the present study was that there was no indication that contralesional flankers affected performance differently from ipsilesional flankers. This pattern suggests that, at least under conditions of central target presentation and target location certainty, pulvinar damage does not necessarily affect flanker processing.

If the pulvinar is involved in attentional selection, why did we not find asymmetric interference effects from contralesional and ipsilesional flankers? One possibility is related to the selection requirements of the task. In Experiment 1 , the target always appeared at fixation and its location was known. It is possible that pulvinar damage affects attentional processing only when the target location is not known in advance and/or when targets appear lateral to the focus of attention/fixation and a shift of attention is necessary. In order to test this possibility, we introduced target location uncertainty in Experiment 2 and varied the target's position relative to the focus of attention. This was done by having the patients respond to a target that could appear at fixation (center), to the left, or to the right. The task was similar to the peripheral report task, which has been employed previously in patients with hemispatial neglect (Cohen et al., 1995) and in patients with lesions of lateral PFC (Rafal et al., 1996), except that a central target condition was included. Note that for central targets, the only difference between Experiments 1 and 2 was that target location was uncertain in the latter, whereas for peripheral targets, the differences between ex- 


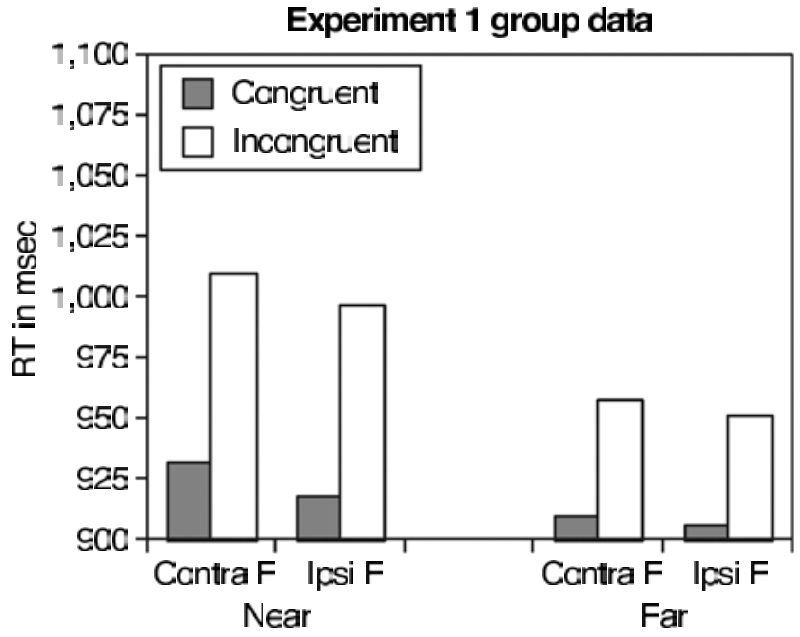

Figure 2. Group reaction times (RTs, in milliseconds) in Experiment 1 as a function of flanker location (contralesional $F$ and ipsilesional F), distance (near and far), and congruency (congruent and incongruent).

periments were both that target location was uncertain and that they appeared lateral to the focus of attention/fixation.

\section{EXPERIMENT 2}

\section{Combined Central and Peripheral Report Task With Manual Responses}

\section{Method}

Procedure. The display, stimulus configurations, and apparatus were identical to those in the near blocks of Experiment 1 except that the target could appear at center, to the left, or to the right. The target was always the smaller of the two squares.

Each patient completed multiple blocks of 128 trials. Within each block, half of the trials were congruent and half were incongruent. On half of the trials the target appeared at center (central targets), and on the remaining trials it appeared with equal probability to the left and right (peripheral targets). On half of the trials the flanker appeared relatively contralesional to the target, and on the remaining trials it appeared relatively ipsilesional to the target. Target color, flanker color, target location, and flanker location were each selected with equal probability, at random and combined orthogonally.

\section{Results}

The data for the group and individual subject analysis were treated in the same way as that in Experiment 1. For each patient, failures to respond in a speeded manner, anticipatory responses, and errors accounted for less than $2 \%$ of the trials.

The mean RTs were entered into a three-way (congruency-congruent and incongruent; target locationcentral and peripheral; and relative flanker locationcontralesional and ipsilesional) repeated measures ANOVA. Figure 3 shows the group RTs for congruent and incongruent trials for central and peripheral targets as a function of relative flanker location.

The group analysis revealed a main effect of congruency $[F(1,2)=517.8, p<.005]$, with RTs shorter on congruent trials $(721 \mathrm{msec})$ than on incongruent trials
$(799 \mathrm{msec})$, and a main effect of target location $[F(1,2)=$ $67, p<.05]$, with shorter RTs on central target trials $(748 \mathrm{msec})$ than on peripheral target trials $(772 \mathrm{msec})$. Most important, congruency interacted with relative flanker location $[F(1,2)=19.5, p<.05]$ in such a way that FCEs were larger when the flanker was relatively ipsilesional than when it was relatively contralesional. An examination of this interaction shows that it is primarily driven by differences in RT performance on the incongruent trials (see Figure 3 for the group data and Table 1 for the individual data). There were no other significant effects.

\section{Discussion}

There were three main findings in this study. First, in a difference from the results of Experiment 1, FCEs were larger on relatively ipsilesional flanker trials than on relatively contralesional flanker trials. Second, although the three-way interaction was not significant, the FCE asymmetry was numerically larger for peripheral targets than for central targets. This latter finding suggests that both target location uncertainty and, potentially, the target's position relative to attention/fixation affects the response activation associated with contralesional stimuli (in Experiment 3, however, the effects for central and peripheral target conditions were very similar). Finally, the difference in RTs for relatively ipsilesional and contralesional flanker conditions was found in the incongruent condition but not in the congruent condition (see Figure 3). The lack of an asymmetric effect in the congruent condition may be explained by the fact that, in this condition, responses can be based on either the target or flanker response activation or on their summed activation. If responses are typically based on the response activation

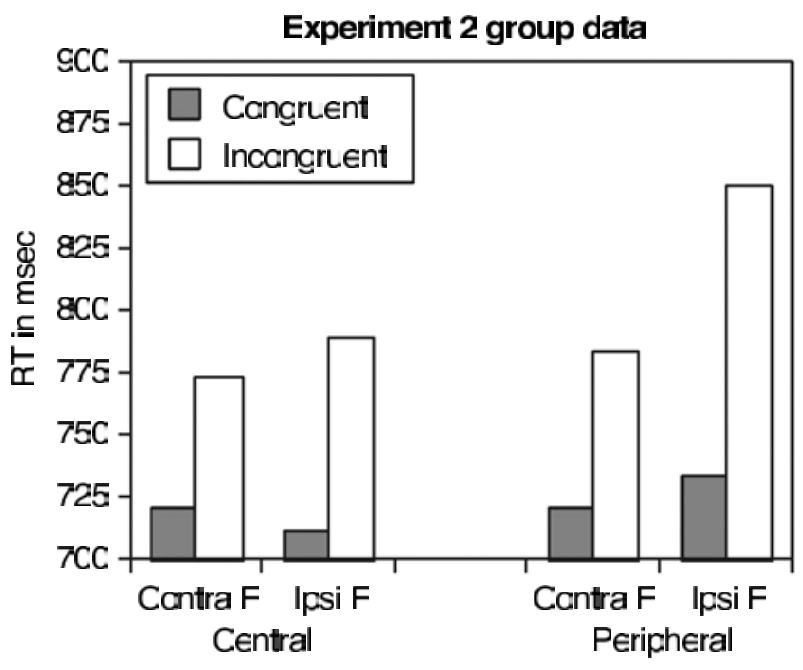

Figure 3. Group reaction times (RTs, in milliseconds) in Experiment 2 as a function of flanker location (contralesional $F$ and ipsilesional F), target location (central and peripheral), and congruency (congruent and incongruent). 
from the relatively ipsilesional stimulus or on the summed activation, flanker-side asymmetric FCEs would not be expected. In contrast, accurate responses in the incongruent condition require subjects to select the response activation associated with the target. If relatively contralesional stimuli produced weaker response activation than ipsilesional stimuli did, one would expect faster RTs for relatively ipsilesional targets. The importance of response competition to impaired contralesional target performance was explored in the next experiment.

\section{EXPERIMENT 3 \\ Combined Central and Peripheral Report Task With Incongruent and Neutral Conditions (Manual Responses)}

In Experiment 3, our aim was to examine whether the RT asymmetry observed on incongruent trials was contingent on response competition from the flanker. To address this question, the congruent condition was replaced with a neutral condition in which the target was paired with a flanker that was not associated with a response. Note that for the neutral condition responses must be based on the response activation associated with the target, whereas in the congruent condition responses may be based on either response activation or their summation (since both indicate the same response). If slowed RTs to contralesional targets are contingent on response competition, then slowed RTs to contralesional targets should be found only in the incongruent trial condition. If, on the other hand, weakened contralesional response activation in the absence of response competition is sufficient to impair performance, then even in the neutral condition contralesional targets should be responded to more slowly than are ipsilesional targets.

\section{Method}

Procedure. The display, stimuli, apparatus, procedure, and design were identical to those used in Experiment 2. The only difference was that the congruent condition was replaced with a neutral condition, in which the green and red targets were paired with a blue flanker. The sizes of the stimuli were the same as those in Experiments 1 and 2 . The target was always the smaller of the two squares.

\section{Results}

The group and individual data analysis was done in the same way as in the previous experiments. For each patient, an inability to respond in a speeded manner, anticipatory responses, and errors accounted for less than $2 \%$ of the trials. The mean RTs were entered into a three-way (congruency-neutral and incongruent; target positioncentral and peripheral; and relative flanker locationcontralesional and ipsilesional) repeated measures ANOVA. Figure 4 shows group incongruent and neutral RTs for central and peripheral targets as a function of relative flanker location.

The group analysis revealed a main effect of congruency $[F(1,2)=83.2, p<.05]$, with RTs shorter on neutral trials $(666 \mathrm{msec})$ than on incongruent trials $(715 \mathrm{msec})$. There

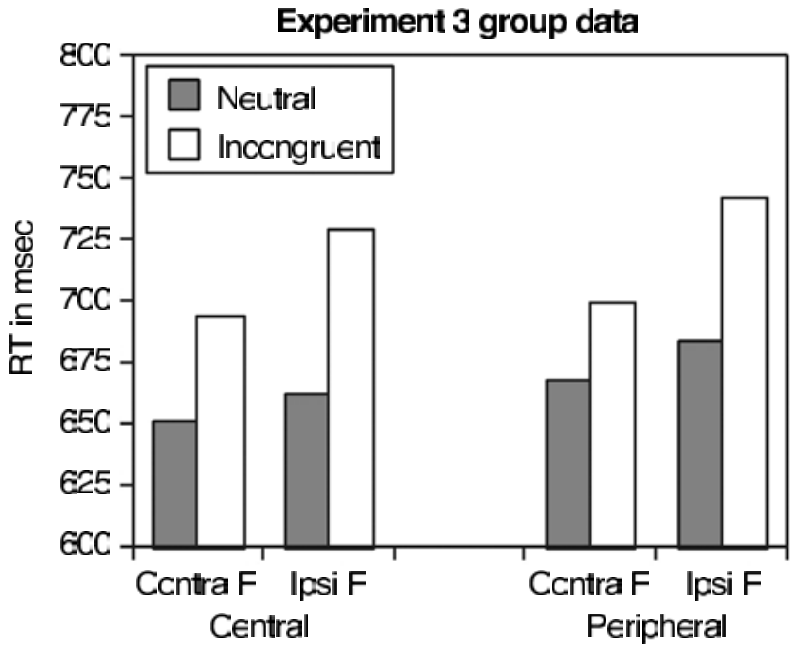

Figure 4. Group reaction times (RTs, in milliseconds) in Experiment 3 as a function of flanker location (contralesional $F$ and ipsilesional F), target location (central and peripheral), and congruency (neutral and incongruent).

was also a main effect of target location $[F(1,2)=126.2$, $p<.01$ ], with RTs on central target trials $(683 \mathrm{msec})$ shorter than those on peripheral target trials $(697 \mathrm{msec})$. Most important, and as in Experiment 2, congruency interacted with relative flanker location $[F(1,2)=202.7$, $p=.005]$ in such a way that, for incongruent trials, responses to targets paired with ipsilesional flankers were slower than were responses to targets paired with contralesional flankers, whereas for neutral trials responses in the two conditions did not differ significantly [T.N., incongruent, $F(1,9)=7.8, p<.05$, and neutral, $F(1,9)<1)$; G.J., incongruent, $F(1,11)=9.5, p<.05$, and neutral, $F(1,9)<1$; S.M., incongruent, $F(1,9)=8.78, p<.05$, and neutral, $F(1,9)=3.84, p=.08]$. There were no other significant effects.

\section{Discussion}

In the present experiment all 3 patients were slower to respond to a relatively contralesional target than to a relatively ipsilesional target in the incongruent condition, whereas only one (S.M.) was marginally slower to respond to such targets in the neutral condition. Thus, all 3 patients demonstrated impaired contralesional performance under conditions of response competition, whereas only one was also impaired when response competition was absent. Thus, in the context of our experiment, response competition was critical for producing a contralesional deficit in performance. This finding, however, does not preclude the possibility that contralesional response activation was weakened. What it does indicate is that contralesional performance is impaired only when relatively ipsilesional stimuli compete for response. Interestingly, and unlike the results from Experiment 2, central and peripheral target performance was indistinguishable. The discrepant findings of the two experi- 
ments make it difficult to conclude whether impaired contralesional processing is contingent on a contralesional shift of attention from fixation.

Independent of the aforementioned claims, the FCE asymmetry found in Experiments 2 and 3 is not consistent with a role for the pulvinar in inhibiting or filtering out irrelevant response activations from contralesional stimuli, since these produced less response activation and not more activation. Furthermore, the data are not consistent with either an engage or disengage deficit, both of which would predict slowing in responses to contralesional and ipsilesional targets in neutral as well as incongruent flanker conditions. The pattern we found in Experiments 2 and 3 is similar to that found by Danziger et al. (2002) in a response competition task with hierarchical figures.

\section{EXPERIMENT 4 Vocal Peripheral Response Task}

In explaining the reduced FCEs from contralesional flankers in patients with PFC damage, Rafal et al. (1996) proposed that the lateral PFC is involved in maintaining arbitrary stimulus-response mappings. On the basis of this hypothesis, they predicted similar FCEs from contralesional and ipsilesional flankers when stimulus-response pairs are not arbitrary, such as when the response required is to say the word "red" in response to a red color patch. In Experiment 4, our aim was to establish whether such a deficit contributed to the asymmetric FCEs observed in Experiments 2 and 3. To this end we evaluated the patients' performance on a vocal response version of the peripheral report task. If a deficit in maintaining stimulus-response mapping contributed to the asymmetric FCEs found in the previous two experiments, these effects should disappear when the task does not require the maintenance of arbitrary mappings.

\section{Method}

Procedure. The patients verbally reported the color of a peripheral small square while ignoring the central large square (flanker). There were no central targets. The display, stimuli, apparatus, procedure, and design were identical to those used in Experiments 2 and 3 and in the central target condition of Experiment 1. Thus, the response was based on an overlearned association between stimulus and response, and there was no requirement to maintain arbitrary stimulus-response mappings in working memory. Verbal RTs were measured using a voice key that was connected to a PsyScope button box. Following the verbal response, an experimenter manually entered the vocal response of the patient.

\section{Results}

The group and single-subject data analysis was the same as that in the previous experiments. For each patient, exclusions on the basis of slow responses and outliers accounted for less than $2 \%$ of the trials. Error rates were very low, with T.N. and G.J. making less than $1 \%$ errors and S.M. making less than 5\% errors.

The mean RTs were entered into a two-way (congruency-congruent and incongruent; and relative flanker

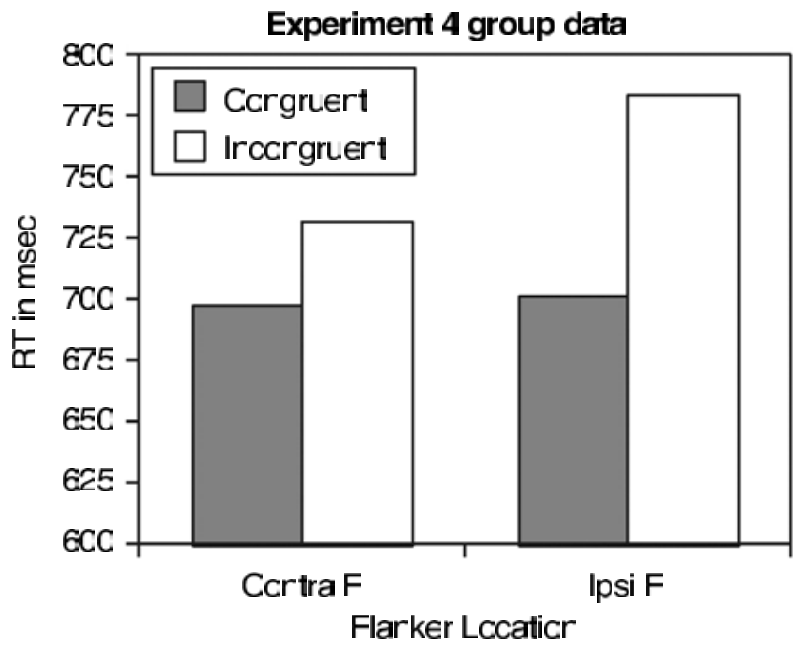

Figure 5. Group reaction times (RTs, in milliseconds) in Experiment 4 as a function of flanker location (contralesional $F$ and ipsilesional F) and congruency (congruent and incongruent).

location-contralesional and ipsilesional) repeated measures ANOVA. Figure 5 shows group FCEs as a function of flanker location. The group analysis revealed a main effect of congruency $[F(1,2)=18.4, p<.05]$, with RTs shorter on congruent trials $(699 \mathrm{msec})$ than on incongruent trials $(757 \mathrm{msec})$. Individual analyses revealed that, for T.N. $[F(1,9)=7, p<.05]$ and S.M. $[F(1,5)=$ $4.8, p<.08$ ], FCEs were larger when the target appeared contralesionally (ipsilesional flanker) than ipsilesionally (contralesional flanker), indicating that the lesion disrupted response activation for nonarbitrary as well as arbitrary stimulus-response mappings in these patients. In contrast, for Patient G.J., FCEs were of equivalent size for contralesional and ipsilesional targets.

\section{GENERAL DISCUSSION}

We evaluated the putative role of the pulvinar in selective attention in 3 patients with unilateral lesions of the posterior thalamus, including the pulvinar. Each patient completed four variants of a flanker interference task in which they reported the color of a square of a specified size while ignoring an irrelevant flanker that was either contralesional or ipsilesional to the target. FCEs were generally larger when the flanker appeared relatively ipsilesionally than contralesionally under target location uncertainty (Experiments 2 and 4). Importantly, the asymmetric FCEs resulted primarily from differences in RTs for the incongruent condition, in which there was response competition. When the flanker was either congruent or neutral (Experiment 3), RTs to ipsilesional and contralesional targets were generally similar. These findings indicate that the pulvinar damage in our patients produced a deficit in contralesional response competition.

Since relatively contralesional flankers, as compared with ipsilesional flankers, did not produce increased in- 
terference, the present findings cannot be explained by accounts that posit a role for the pulvinar in filtering out or inhibiting irrelevant flankers. Furthermore, our findings cannot be explained by a deficit produced by pulvinar damage in either disengaging or engaging visual attention. Most telling in this respect was that in the neutral condition of Experiment 3 we did not find evidence for longer RTs on contralesional target trials than on ipsilesional target trials for 2 of the 3 patients (S.M. being the exception). This is not the first time that we failed to find evidence for an engage deficit in pulvinar patients. In a previous study Danziger et al. (2002) evaluated target selection in a hierarchical figure response competition task. In that experiment there were no differences in overall RT to contralesional and ipsilesional targets, nor was there any evidence for an FCE field asymmetry effect. At present we do not know what underlying difference between the spatial cuing task, which produces an engage-like deficit, and the two-choice discrimination task, which does not, causes these differences. Some potential candidates may be the difference in response selection requirements (e.g., spatial precuing, simple detection, response competition, two choice) or differences in temporal parameters (e.g., cue and target appear successively in the cuing tasks whereas the target and flankers appear simultaneously in the response selection tasks that we have used).

We will first discuss our observations in light of the literature from animal experimentation and human brain activation studies. We will then discuss our observations in relation to the observations reported previously in studies that used a similar flanker paradigm in patients with hemispatial neglect and patients with lesions of the PFC.

\section{Convergence With Animal Experimentation}

A series of studies has explored the role of the pulvinar in selective processing in nonhuman primates (Desimone et al., 1990; Petersen, Robinson, \& Keys, 1987; Petersen, Robinson, \& Morris, 1987). Physiological recordings have demonstrated changes in neuronal activity when a monkey attends to a task-relevant stimulus (e.g., Petersen, Robinson, \& Keys, 1987). As with any activation method, in which behavior can be correlated only with neuronal activity, such observations cannot specify the function of the pulvinar: They do not necessarily indicate that the pulvinar is crucial for allocating attention or, if it is, whether it functions to enhance perceptual processing or response selection. Petersen, Robinson, and Morris transiently inactivated the pulvinar and confirmed Rafal and Posner's (1987) findings in neurological patients that responses to contralesional targets in a spatial precuing task were delayed.

The most relevant of the physiological studies in nonhuman primates to the subject of our investigation was a study by Desimone et al. (1990). In this study, monkeys were trained to discriminate color and form targets in the presence of distracting visual information until performance reached ceiling. At this point, the pulvinar was inactivated by injections of muscimol. Desimone et al. found that when a target was presented alone, error rates in both visual fields were similar $(\sim 10 \%)$. In contrast, when the target appeared simultaneously with a distractor in the opposite visual field (each stimulus $3^{\circ}$ from fixation), error rates were significantly higher when the target appeared in the contralesional field ( $30 \%$ errors) than when it appeared in the ipsilesional field $(\sim 10 \%$ errors). These error rate results are similar to our RT results. In both studies, contralesional targets were affected more strongly by ipsilesional distracting information than were ipsilesional targets by contralesional distracting information. Desimone et al.'s results have previously been interpreted as a case in which pulvinar damage induces a competitive bias against the allocation of spatial attention to the contralesional field (Duncan, Humphreys, \& Ward, 1997). As was noted above, our data are not consistent with a general engage or disengage deficit. Instead, we suggest that the results from both Desimone et al. and the present study are consistent with an account whereby some aspect of ipsilesional stimulus processing is favored over contralesional stimulus processing. Our results suggest that pulvinar damage produces a contralesional deficit in response competition.

\section{Comparison With Previous Patient Studies of Flanker Interference}

We have argued that the asymmetric FCEs observed in our patients can be attributed to lesions of the thalamus. Although for all 3 patients the main damage was to the pulvinar, it is also almost certainly the case that the reticular nucleus of the thalamus (which invests the pulvinar and other thalamic nucleii) was compromised as well. We cannot conclude definitively, therefore, that pulvinar damage is both necessary and sufficient to impair response activation and selection of contralateral stimuli, nor can we exclude a role for the reticular nucleus or other nuclei of the posterior thalamus in these processes.

\section{Comparison of Parietal- and Pulvinar-Lesioned Patients}

It has recently been confirmed by Karnath, Himmelbach, and Rorden (2002) that acute lesions of the pulvinar can produce hemispatial neglect. They noted that neglect after pulvinar lesions was ty pically resolved within a few weeks or months, and they attributed this neglect to diaschesis that compromises the function of the superior temporal gyrus, rather than to the pulvinar's role in the orientation of visual attention. The results from our patients, collected many months after their initial trauma, confirm this account of Karnath et al.'s findings. None of the patients in the present study, at the time of testing, showed any clinical signs of hemispatial neglect or inattention. Moreover, as will be discussed next, none showed an RT deficit similar to those of patients with hemispatial neglect tested in a similar paradigm.

Parietal-lesioned patients have been tested in two studies by using a task similar to that used here. In the first of 
the two studies, which was most similar to ours, Cohen et al. (1995) tested 2 patients with hemispatial neglect on central and peripheral report tasks. There were two main findings. First, both patients responded more slowly to central targets when there was an ipsilesional flanker, regardless of whether it was congruent or incongruent. Second, FCEs were equivalent whether the flanker was relatively ipsilesional or contralesional, regardless of whether the target was presented in a central or peripheral location. In the second study, Ro et al. (1998) tested 12 patients with posterior parietal lobe damage, 6 with temporoparietal involvement and 6 without (most of the patients did not suffer from hemispatial neglect). This study differed from Cohen et al.'s and our study in that each patient performed only 96 trials, targets always appeared centrally, and flankers were presented for only 16.7 msec. Unlike Cohen et al., Ro et al. found that patients with temporoparietal involvement showed no interference effects from contralesional flankers and were no slower to respond to the target regardless of whether the flanker was ipsilesional or contralesional. In a followup study aimed at understanding the discrepancy in the results between the two studies, Ro et al. found that when temporoparietal patients performed many more trials, the results became similar to those of Cohen et al. Nevertheless, Ro et al.'s main conclusion was that the temporoparietal junction is involved in transducing perception into action. Importantly, the results of our study differ from the observations of Cohen et al. and Ro et al. (in the extensive training case). In our case, central target performance was not impaired when target location was certain, nor was there evidence for a disengage deficit from ipsilesional stimuli.

We can also compare the effects of flanker interference in pulvinar and parietal patients. The hemispatial neglect patients reported by Cohen et al. (1995) and the 2 patients that underwent extensive testing in Ro et al.'s (1998) study showed FCEs that were equivalent whether the flanker was relatively ipsilesional or contralesional, regardless of whether the target was presented in a central or peripheral location. In contrast, our patients had asymmetric FCEs when target location was not known.

On the basis of these findings, we can provisionally conclude that lesions to PAC produce a pattern consistent with attentional capture by relatively ipsilesional stimuli and a deficit in orienting contralesionally, but they do not affect the processing of the flanker or its activation of competing response channels (at least with extensive testing). By contrast, pulvinar lesions lead to a contralesional deficit in response competition.

This dissociation in patients demonstrates that the FCE is not necessarily reduced under conditions of inattention and that it can provide a measure of response selection that is independent from the locus of spatial attention. Ro, Machado, Kanwisher, and Rafal (2002) have recently provided further evidence for the independence of the FCE and the locus of spatial attention in a study in normal participants. Using a paradigm similar to that employed here, they showed that the FCE was not modulated by the allocation of attention, either when deployed voluntarily or summoned reflexively.

\section{Comparison of Prefrontal- and Pulvinar- Lesioned Patients}

Rafal et al. (1996) tested 10 patients with unilateral lesions of lateral PFC with the use of tasks similar to those used here. The main finding of this study was that FCEs from contralesional flankers were reduced both in a central report task (like in the present Experiment 1, in which pulvinar-lesioned patients showed no asymmetry in FCEs) and in a peripheral report keypress task. On the basis of these findings, Rafal et al. suggested that the PFC plays a role in maintaining the arbitrary mappings between color and keypress responses and that the asymmetric FCEs resulted from a failure of contralesional stimuli to be linked to their arbitrary responses (whether they were a target or a flanker). A subsequent study of these patients, in which verbal naming responses were made (like in the present Experiment 4), showed similar FCEs in the contralesional and ipsilesional fields (Henik, Ro, Rafal, \& Egly, 1998). This pattern was the same as that shown by Patient G.J., but not by Patient S.M. or T.N., in our study. Thus, although both frontal and pulvinar lesions can affect FCEs, we suggest that they do so by interfering with different stages of processing. The pulvinar appears to be involved in response activation and selection linking stimuli to response, and the PFC is required when this mapping is arbitrary and requires maintenance of stimulus-response mappings in working memory.

\section{REFERENCES}

Cohen, A., Ivry, R., RAFAL, R. D., \& KoHn, C. (1995). Response code activation by stimuli in the neglected visual field. Neuropsychology, 9, $165-173$.

Coles, M. G., Gratton, G., Bashore, T. R., Eriksen, C. W., \& DoNCHIN, E. (1985). A psychophysiologicalinvestigation of the continuous flow model of human information processing. Journal of Experimental Psychology: Human Perception \& Performance, 11, 529553.

DANZIGER, S., WARD, R., OWEN, V., \& RAFAL, R. D. (2002). The effects of unilateral pulvinar damage in humans on reflexive orienting and filtering of irrelevant information. Behavioral Neurology, 13, 95-104.

Desimone, R., Wessinger, M., Thomas, L., \& Schneider, W. (1990). Attentional control of visual perception: Cortical and subcortical mechanisms. Cold Spring Harbor Symposia on Quantitative Biology, 55, 963-971.

Diedrichsen, J., Ivry, R. B., Cohen, A., \& DAnziger, S. (2000). Asymmetries in a unilateral flanker task depend on the direction of the response: The role of attentional shift and perceptual grouping. Journal of Experimental Psychology: Human Perception \& Performance, 26, 113-126.

DunCAN, J., Humphre Ys, G. W., \& WARd, R. (1997). Competitive brain activity in visual attention. Current Opinion in Neurobiology, 7, 255261.

ERIKSEN, C. W., \& YEH, Y. (1985). Allocation of attention in the visual field. Journal of Experimental Psychology: Human Perception \& Performance, 11, 583-597.

Friedrich, F. J., EGLY, R., RAFAL, R. D., \& BECK, D. (1998). Spatial attention deficits in humans: A comparison of superior parietal and temporal-parietal junction lesions. Neuropsychology, 12, 193-207. 
HENIK, A., Ro, T., RAFAL, R. D., \& EGLY, R. (1998). Involvement of the lateral prefrontal cortex in color-word interactions. Journal of the International Neuropsychological Society, 4, 222.

Karnath, H. O., Himmelbach, M., \& Rorden, C. (2002). The subcortical anatomy of human spatial neglect: Putamen, caudate nucleus and pulvinar. Brain, 125, 350-360.

LABERGE, D. (1990). Thalamic and cortical mechanisms of attention suggested by recent positron emission tomographic experiments. Journal of Cognitive Neuroscience, 2, 358-372.

LaBerge, D., \& Buchsbaum, M. S. (1990). Positron emission tomographic measurements of pulvinar activity during an attention task. Journal of Neuroscience, 10, 613-619.

LAVIE, N. (1995). Perceptual load as a necessary condition for selective attention. Journal of Experimental Psychology: Human Perception \& Performance, 21, 451-468.

MiLler, J. (1991). The flanker compatibility effect as a function of visual angle, attentional focus, visual transients, and perceptual load: A search for boundary conditions. Perception \& Psychophysics, 49, 270-288.

Morris, J. S., Friston, K. J., \& DolAn, R. J. (1997). Neural responses to salient visual stimuli. Proceedings of the Royal Society of London: Series $B, \mathbf{2 6 5}, 769-775$.

Morrow, L. A., \& Ratcliff, G. C. P. (1988). The disengagement of covert attention and the neglect syndrome. Psychobiology, 16, 261269.

Petersen, S. E., Robinson, D. L., \& Keys, W. (1987). Pulvinar nuclei of the behaving rhesus monkey: Visual responses and their modulation. Journal of Neurophysiology, 54, 867-886.

Petersen, S. E., Robinson, D. L., \& Morris, J. D. (1987). Contributions of the pulvinar to visual spatial attention. Neuropsychologia, 25, 97-106.

Posner, M. I., \& Petersen, S. E. (1990). The attention system of the human brain. Annual Review of Neuroscience, 13, 25-42.

Posner, M. I., WALKer, J. A., Friedrich, F. J., \& RAFAL, R. (1984). Effects of parietal injury on covert orienting of visual attention. Journal of Neuroscience, 4, 1863-1874.
Rafal, R. [D.], Gershberg, F., Egly, R., Ivry, R., Kingstone, A., \& Ro, T. (1996). Response channel activation and the lateral prefrontal cortex. Neuropsychologia, 34, 1197-1202.

RAFAL, R. D., \& POSNER, M. I. (1987). Def icits in human visual spatial attention following thalamic lesions. Proceedings of the National Academy of Sciences, 84, 7349-7353.

Ro, T., Cohen, A., Ivry, R. B., \& RAFAL, R. D. (1998). Response channel activation and the temporo-parietal junction. Brain \& Cognition, 37, 461-476.

Ro, T., MAchado, L., Kanwishe R, N., \& RAFAL, R. D. (2002). Covert orienting to the locations of targets and distractors: Effects of response channel activation in a flanker task. Quarterly Journal of Experimental Psychology, 55, 917-936.

Robinson, D. L., \& Petersen, S. E. (1992). The pulvinar and visual salience. Trends in Neurosciences, 15, 127-132.

SAPIR, A., RAFAL, R. D., \& HENIK, A. (2002). Attending to the thalamus: Inhibition of return and nasal-temporal asymmetry in the pulvinar. NeuroReport, 13, 693-697.

Sturm, W., de Simone, A., Krause, B. J., Specht, K., Hesselmann, V., Radermacher, I., Herzog, H., Tellmann, L., Müller-Gärtner, H.-W., \& WiLLMES, K. (1999). Functional anatomy of intrinsic alertness: Evidence for a fronto-parietal-thalamic-brainstem network in the right hemisphere. Neuropsychologia, 37, 797-805.

WARD, R., DANZIGER, S., OWE N, V., \& RAFAL, R. D. (2002). Deficits in spatial coding and feature binding following damage to the human pulvinar. Nature Neuroscience, 5, 99-100.

\section{NOTE}

1. In Experiment 3, the congruent condition was replaced with a neutral condition, in which the flanker was not associated with a potential response.

(Manuscript received June 11, 2003; revision accepted for publication February 25, 2004.) 